\title{
Mineração
}

\section{Um modelo de programação matemática para otimizar a composição de lotes de minério de ferro da mina Cauê da CVRD}

\author{
Edilaila Fernandes Moraes \\ Mestre em Eng. Mineral - PPGEM/EM/UFOP - Ouro Preto - MG.E-mail: edilaila.moraes@cvrd.com.br \\ José Maria do Carmo Bento Alves \\ Mestrando - PPGEM/EM/UFOP - Ouro Preto -MG.E-mail: jmalves@uai.com.br \\ Marcone Jamilson Freitas Souza \\ Prof. Adjunto - PPGEM/EM/UFOP e DECOM/ICEB/UFOP - Ouro Preto - MG \\ E-mail:marcone@iceb.ufop.br \\ Ivo Eyer Cabral \\ Prof. Adjunto - PPGEM/EM/UFOP e DEMIN/EM/UFOP - Ouro Preto \\ MG.E-mail:cabralmg@uai.com.br \\ Alexandre Xavier Martins \\ Prof. Assistente -DECEA/UFOP - Ouro Preto - MG.E-mail:xmartins@decea.ufop.br
}

\section{Resumo}

Esse trabalho tem seu enfoque no problema de blendagem de produtos de minério de ferro, estocados nos pátios da mina Cauê, da Companhia Vale do Rio Doce, em Itabira, Minas Gerais, para a composição de lotes. Propõe-se um modelo de programação linear por metas que visa a determinar os locais de retomada dos produtos estocados, de tal forma que a mistura atenda aos limites de especificações de qualidade e quantidade preestabelecidos pelo cliente e satisfaça as restrições operacionais dos pátios. O modelo de programação matemática desenvolvido foi implementado no modelador e otimizador LINGO 9.0, interfaceando com planilhas do EXCEL 2000, possibilitando a utilização e exportação de dados em um ambiente familiar à empresa de mineração. O sistema desenvolvido foi validado comparando-se os resultados obtidos com os produzidos manualmente pela empresa. Os resultados computacionais apresentados comprovaram que é possível prover uma melhora na composição dos lotes através do modelo proposto.

Palavras-chave: Blendagem de minérios, programação linear por metas, pátio de estocagem de minérios.

\section{Abstract}

This work focuses on the problem of blending iron ore products, at the Cauê mine stockyard, of Companhia Vale do Rio Doce, in the state of Minas Gerais, for the formation of lots. It consists in elaborating a linear goal programming model that seeks to determine the areas to recapture the stored ore in such a way that the blending of products be in conformity with the quality specification required by the customer. This work is a case study of an applied nature. The mathematical programming model was developed with the support of the optimization software LINGO 9.0 in conjunction with EXCEL 2000 spreadsheets, making it possible to handle and export data in formats used by the mining company. With the purpose of validating the implemented system, the results obtained by the system were compared with real data. These results proved that is possible to improve the composition of the product lots applying the proposed model.

Keywords: Ore blending, goal programming, ore stockyard. 


\section{Introdução}

Em busca de competitividade e mesmo de sobrevivência, as empresas buscam otimizar seus processos produtivos, gerenciando, eficientemente, seus recursos disponíveis. Nesse contexto, a Pesquisa Operacional tornou-se ferramenta muito importante no meio empresarial, facilitando a otimização e racionalização de tais recursos. Isso se deve à característica principal dos modelos de otimização de indicarem a melhor decisão a ser tomada segundo algum critério adotado.

As técnicas de modelagem matemática ou de otimização geralmente estudadas estão condicionadas à solução de um único objetivo e assumem que as restrições não podem ser violadas.

Entretanto inúmeras soluções existentes no mundo real exigem que boa parte das decisões do dia-a-dia das empresas seja flexível. Dessa forma, os gestores procuram satisfazer ou pelo menos se aproximar dos objetivos estabelecidos, em vez de considerá-los como rígidos.

Problemas dessa natureza podem ser mais apropriadamente modelados utilizando-se múltiplos objetivos em vez de restrições rígidas. A solução desses modelos não se resume a maximizar ou minimizar uma função-objetivo satisfazendo a todo um conjunto de restrições, mas envolve a satisfação de uma condição mínima aceitável. Esses são os denominados problemas de programação por metas, conhecidos na literatura inglesa como Goal Programming (Bueno e Oliveira, 2004).

A programação por metas é uma técnica que permite a modelagem e a busca de soluções para os problemas com múltiplos objetivos ou metas a serem otimizadas. Uma das diferenças significativas entre ela e a programação linear clássica está na função-objetivo. Esta última requer que o tomador de decisão persiga apenas uma função-objetivo: a minimização de custos, ou a maximização do lucro ou qualquer outra função a ser otimizada. Na programação por metas, por outro lado, não se busca so- mente maximizar ou minimizar a funçãoobjetivo diretamente; mas, também, a minimização dos desvios no alcance das metas. Para esse fim, são utilizadas as chamadas variáveis de desvio, as quais medem a "distância" entre os objetivos estabelecidos.

Nessas condições, a solução ideal seria aquela na qual todas as variáveis de desvio tivessem valor igual a zero, ou seja, em que todos os objetivos tivessem sido atingidos em seus valores estipulados.

Dessa forma, para a solução dos problemas de programação por metas, devem ser identificadas, além das variáveis de decisão presentes nos modelos de programação linear, as respectivas variáveis de desvio.

Esse trabalho aborda o problema dos pátios de estocagem de minérios através de programação linear por metas arquimediana, nos termos de Romero (2004). Um modelo voltado para a mineração, descrito em Chanda e Dagdelen (1995), contempla a resolução de um problema similar com o atendimento às metas de qualidade, mas não o seqüenciamento das máquinas retomadoras. Ademais, faz-se necessário incluir, nesse modelo, várias outras restrições operacionais. Por exemplo, determinadas áreas dos pátios devem ser estimuladas a serem esvaziadas periodicamente com vistas à reposição de material. Pilhas “distantes" umas das outras não podem ser retiradas em uma mesma operação, devido ao tempo de deslocamento das máquinas retomadoras e o curto tempo disponível para a composição de um lote de vagões.

Esse trabalho está organizado como segue. Na seção 2, são apresentados os conceitos de blendagem e homogeneização. Na seção 3, o problema dos pátios de estocagem de produtos da Companhia Vale do Rio Doce é descrito. Na seção 4, apresenta-se o modelo de programação linear por metas para resolver o problema abordado. Os resultados obtidos pela aplicação do modelo são apresentados e discutidos na seção 5 , enquanto a última seção conclui o trabalho.

\section{Conceitos de blendagem e homogeneização}

Um problema muito comum em minas a céu aberto que operam com diversas frentes de lavra simultâneas é determinar o ritmo de lavra de cada frente para que uma blendagem ou mistura de minérios seja feita de maneira a satisfazer as exigências de qualidade e quantidade de um cliente, já que cada frente de minério possui características químicas e granulométricas diferentes.

O minério proveniente dessas frentes pode servir de alimentação para uma usina de concentração. Essa usina, para operar de um modo eficiente, sem que haja necessidade de constantes ajustes operacionais, requer uma alimentação mais homogênea que apresente pequenas flutuações nas suas características físicas e químicas. O minério, antes de alimentar a usina, pode ser depositado em um pátio de homogeneização na forma de pilhas, que, quando formadas e retomadas, adequadamente, produzem a requerida homogeneização. Dessa forma, a alimentação da usina apresentará menores flutuações ou desvios dos parâmetros de qualidade em relação a valores médios ou metas, quando comparadas com as flutuações presentes no minério antes da homogeneização.

O termo blendagem diz respeito a uma mistura, em proporções definidas, de minérios de características diferentes, com o objetivo de se obter uma massa com características específicas.

O termo homogeneização se refere ao manuseio ou mistura de quantidades de minério, com o objetivo de se obter um conjunto que tenha composição ou características uniformes.

Deve-se ressaltar que o conceito de um conjunto homogêneo está sempre vinculado a uma escala de observação ou a um tamanho de amostra de referência, ou seja, um conjunto será considerado homogêneo, se não há significantes diferenças nas características médias de qualquer parte do conjunto, desde 
que a amostra de observação seja de tamanho igual ao de referência.

Um estudo detalhado dos conceitos de blendagem e homogeneização pode ser encontrado em Schofield (1980).

Uma outra situação de mistura de minérios que pode ocorrer numa mineração e na qual se insere esse trabalho é quando produtos com parâmetros de qualidades diferentes são depositados na forma de pilhas em um pátio de estocagem e precisam ser misturados para se obter um lote de carregamento de vagões de minério que atenda aos limites de especificações de qualidade e de quantidade de um dado cliente.

\section{Descrição do problema dos pátios de estocagem de produtos}

Esse trabalho tem seu enfoque nos pátios de estocagem da mina Cauê, da Companhia Vale do Rio Doce (CVRD), em Itabira (MG). As minas de Itabira são responsáveis por $50 \%$ de toda a produção do Sistema Sul (CVRD, 2003), formado pelas minas do Quadrilátero Ferrífero.

Minérios de diferentes especificações, sejam elas químicas (teores de ferro, sílica, manganês, etc.), ou físicas (no caso, granulometria), são armazenados na forma de pilhas em 3 (três) pátios de estocagem para posterior retomada visando à composição de lotes de vagões, em atendimento às encomendas de clientes. Para esse específico problema, o termo pilhas se refere aos minérios ou produtos armazenados nos pátios em determinados espaços graduados de 5 em 5 metros. Esses espaços recebem o nome de balizas.

Para a formação de um lote de vagões, é necessário observar vários aspectos. Do ponto de vista da qualidade do minério, para cada parâmetro de qualidade, o cliente especifica as metas, bem como os limites mínimos e máximos toleráveis. Do ponto de vista operacional, as máquinas retomadoras de minério têm suas limitações. A retomada de minério é sempre feita em um mesmo pátio da esquerda para a direita. Assim, para pilhas dispostas em forma contígua, não é possível retirar o minério das pilhas intermediárias sem que as pilhas mais externas à esquerda tenham sido retiradas. Em cada baliza, são armazenados minérios com até duas qualidades diferentes, de forma que a retomada se dá em dois níveis. Pela geometria da formação das pilhas, não é possível retirar o minério situado na parte inferior de uma baliza, se o minério situado na parte superior da baliza à sua direita não tiver sido retirado. Há, também, a limitação de tempo para a composição de um lote. Devido ao tempo de preparação das máquinas retomadoras, não é viável manusear pilhas distantes umas das outras em um mesmo pátio.

Atualmente a programação de lotes é feita manualmente, com auxílio de uma planilha eletrônica. O operador dessa ferramenta simula vários cenários, com base na tentativa e erro, escolhendo aquele que melhor se aproxima do pedido do cliente. A otimização do processo é, portanto, tímida e está baseada apenas na experiência do operador. Dessa forma, o presente trabalho se justifica, uma vez que permitirá obter a melhor solução existente para uma dada configuração de pátio, além de acelerar o processo de tomada de decisão.

É importante observar que, embora esse trabalho esteja voltado para um estudo de caso, toda mineradora tem um pátio de estocagem e os problemas são bastante semelhantes. Assim, a solução a ser adotada para o problema objeto de estudo pode ser utilizada e/ou adaptada para resolver problemas similares de outras empresas mineradoras.

\section{Modelo de programação matemática}

O modelo de programação linear por metas apresentado a seguir é baseado nos dados do Controle de Qualidade da Mina de Cauê da CVRD e visa ao melhor aproveitamento dos recursos e conse- qüente melhoria na produtividade do processo.

\subsection{Parâmetros do modelo}

Sejam os seguintes parâmetros de entrada:

Pátio: Conjunto de pátios, no caso, Pátio $=\{A, B, C\}$.

Baliza (k): Conjunto de balizas do pátio $k$. Para cada pátio $k$, o número de balizas $n b$ varia no intervalo $[1, n b(k)]$. Para o pátio A, tem-se: Baliza $(\mathrm{A})=\{-$ $25,-20,-15, \ldots, 330\}$, isto é, $n b(A)=71$.

Cada pilha situada em uma dada baliza $i$ de um dado pátio $k$ é dividida em duas partes: uma superior, denotada por sup, e outra inferior, denotada por inf.

Retomar $_{i j k}$ : Parâmetro que assume o valor 1 , se a pilha situada na baliza $i$, parte $j$ do pátio $k$, deve ser completamente retomada, como desejo do operador.

$\operatorname{RetParc}_{i j k}$ : Quantidade de minério, em toneladas, a ser retomado da parte $j \mathrm{da}$ baliza $i$ do pátio $k$.

$Q u_{i j k}$ : Quantidade de minério, em toneladas, existente na baliza $i$ do pátio $k$, na parte $j$.

tem $_{i j k}$ : Parâmetro que assume valor 1 , se há um conjunto de pilhas contíguas iniciando na baliza $i$, parte $j$, do pátio $k$. Para as demais balizas, nas quais não há uma pilha à sua esquerda, esse parâmetro assume o valor zero.

$n c p_{k}$ : Número máximo de conjuntos de pilhas contíguas a serem retiradas do pátio $k$ nas posições inf e sup de cada pilha.

$S$ : Conjunto dos parâmetros de qualidade analisados no minério.

$t_{i j k l}$ : Teor do parâmetro $l$ na parte $j$ da pilha situada na baliza $i$ do pátio $k(\%)$.

tr: : Teor recomendado para o parâmetro $l$ no produto final (\%).

lig: Limite inferior de garantia, isto é, teor mínimo admissível para o parâmetro $l$ no produto final (\%). 
$l s g_{l}$ : Limite superior de garantia, isto é, teor máximo admissível para o parâmetro $l$ no produto final (\%).

$l i e_{l}$ : Limite inferior de especificação, isto é, teor mínimo recomendável para o parâmetro $l$ no produto final (\%).

Ise $e_{\text {: }}$ Limite superior de especificação, isto é, teor máximo recomendável para o parâmetro $l$ no produto final (\%).

$\alpha_{1}^{-}$: Penalidade por desvio negativo em relação à meta de qualidade para o parâmetro $l$ no produto final.

$\alpha_{1}^{+}$: Penalidade por desvio positivo em relação à meta de qualidade para o parâmetro $l$ no produto final.

$\gamma_{1}^{+}$: Penalidade por desvio positivo em relação ao limite superior de especificação do parâmetro $l$ no produto final.

$\gamma_{1}^{-}$: Penalidade por desvio negativo em relação ao limite inferior de especificação do parâmetro $l$ no produto final.

$\varphi_{1}^{+}$: Penalidade por desvio positivo em relação ao limite superior de garantia do parâmetro $l$ no produto final.

$\varphi_{1}^{-:}$: Penalidade por desvio negativo em relação ao limite inferior de garantia do parâmetro $l$ no produto final.

$\beta$ : Penalidade por desvio negativo na meta de produção.

$\beta^{+}$: Penalidade por desvio positivo na meta de produção.

$P_{m}$ : meta de produção, em toneladas.

\subsection{Variáveis de decisão}

O modelo proposto considera as seguintes variáveis de decisão:

$X_{i j k}=$ Quantidade de minério, em toneladas, a ser retomado da parte $j$ da baliza $i$ do pátio k.

$y_{i j k}= \begin{cases}1 & \text { se a pilha da baliza } i, \text { pátio } k \text {, parte } j \text {, pode ser usada } \\ 2 & \text { caso contrário }\end{cases}$

$z_{i j k}= \begin{cases}1 & \text { se a pilha da baliza } i, \text { pátio } k \text {, parte } j \text {, será usada } \\ 2 & \text { caso contrário }\end{cases}$

$d_{1}^{-=}$Desvio negativo, em toneladas, do parâmetro $l$ no produto final, em relação à meta de qualidade do parâmetro $l$.

$d_{1}^{+}=$Desvio positivo, em toneladas, do parâmetro $l$ no produto final, em relação à meta de qualidade do parâmetro $l$.

$d_{b a l i z a}{ }_{i j k}=$ Quantidade de minério que resta na pilha localizada na baliza $i$, parte $j$ e pátio $k$.

$P^{-}=$Desvio negativo em relação à meta de produção, em toneladas.

$P^{+}=$Desvio positivo em relação à meta de produção, em toneladas.

$d e_{1}^{+}=$Desvio positivo de especificação do parâmetro $l$ no produto final, em toneladas, em relação ao seu limite superior de especificação.

$d e_{1}^{-}=$Desvio negativo de especificação do parâmetro i no produto final, em toneladas, em relação ao seu limite inferior de especificação.

$d g_{1}{ }^{+}=$Desvio positivo de garantia do parâmetro $l$ no produto final, em toneladas, em relação ao seu limite superior de garantia.

$d g_{1}^{-}=$Desvio negativo de garantia do parâmetro $l$ no produto final, em toneladas, em relação ao seu limite inferior de garantia.

\subsection{Função-objetivo}

A função-objetivo considerada visa a minimizar os desvios de qualidade e quantidade em relação às metas de qualidade e produção, bem como retomar dos pátios, sempre que possível, as pilhas que forem usadas para a composição de um lote:

$$
\begin{gathered}
\min \sum_{l \in S} \alpha_{l}^{-} d_{l}^{-}+\sum_{l \in S} \alpha_{l}^{+} d_{I}^{+}+\beta^{-} P^{-}+\beta^{+} P^{+}+\sum_{l \in S} \gamma_{l}^{+} d e_{I}^{+}+\sum_{l \in S} \gamma_{l}^{-} d e_{I}^{-}+ \\
\sum_{l \in S} \varphi_{l}^{-} d g_{I}^{-}+\sum_{l \in S} \varphi_{I}^{+} d g_{I}^{+}+\sum_{i \in \text { Baliza }} \sum_{j \in \text { Parte }} \sum_{k \in \text { Patio }} d b a l i z a_{i j k}
\end{gathered}
$$




\subsection{Restrições de seqüenciamento vertical}

As equações (2) e (3) modelam a restrição de que só se pode retomar minério da parte inferior de uma pilha, se o minério da parte superior da pilha subseqüente já tiver sido retirado:

$y_{i, \text { inf }, k} \leq y_{i+1, \text { sup }, k} \quad \forall i \in \operatorname{Baliza}(k), \forall k \in$ Pátio, tq $i \leq \mathrm{nb}(k)-1$

$y_{i, \text { inf }, k} \leq \frac{x_{i+1, \text { sup }, k}}{Q u_{i+1, \text { sup }, k}} \forall i \in \operatorname{Baliza}(k), \quad \forall k \in$ Pátio, tq $\mathrm{Qu}_{i+1, \text { sup }, k} \neq 0$ e $i \leq \operatorname{nb}(k)-1$ (3)

\subsection{Restrições para forçar retomada concentrada}

Para cada pátio k, pode ser desejável, por questões operacionais, retomar minério de forma concentrada em um ou mais conjuntos contíguos de pilhas. As equações a seguir modelam essa restrição:

$\sum_{i \in \operatorname{Baliza}(k)}$ tem $_{i, \text { sup }, k} z_{i, \text { sup }, k}+\sum_{i \in \operatorname{Baliza}(k)}$ tem $_{i, \text { inf }, k} z_{i, \text { inf }, k} \leq n c p_{k} \quad \forall k \in$ Pátio, $\forall i \in \operatorname{Baliza}(k)$

$z_{i j k} \leq x_{i j k} \quad \forall k \in$ Pátio, $\forall j \in$ Parte, $\forall i \in \operatorname{Baliza}(k)$

$z_{i j k} \geq \frac{x_{i j k}}{Q u_{i j k}} \quad \forall k \in$ Pátio, $\forall j \in$ Parte, tq $Q u_{i j k} \neq 0$

\subsection{Restrições de seqüenciamento horizontal}

Uma parte $j$ de uma pilha situada na baliza $i$ do pátio $k$ só pode ser retomada, se a mesma parte $j$ da pilha situada na baliza anterior do mesmo pátio $k$ já tiver sido retomada:

$\begin{array}{ll}y_{i j k} \leq \frac{x_{i-1, j, k}}{Q u_{i-1, j, k}} & \forall i \in \operatorname{Baliza}(k), \forall j \in \text { Parte, } \forall k \in \text { Pátio, tq } i \geq 2 \\ y_{i j k} \leq \frac{x_{i j k}}{Q u_{i j k}} & \forall i \in \operatorname{Baliza}(k), \forall j \in \text { Parte, } \forall k \in \text { Pátio, tq } Q u_{i j k} \neq 0\end{array}$

\subsection{Restrições de limite superior de garantia}

As restrições (9) definem limites máximos para os parâmetros de qualidade da mistura.

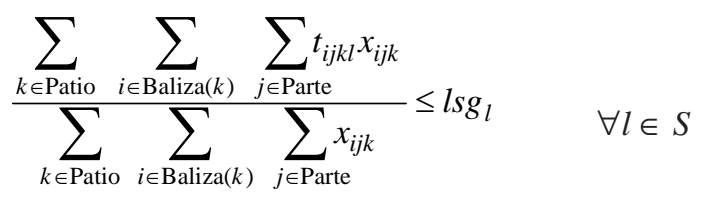

Linearizando e introduzindo variáveis de desvio positivo de garantia, tem-se:

$$
\sum_{k \in \operatorname{Patio}} \sum_{i \in \operatorname{Baliza}(k)} \sum_{j \in \operatorname{Parte}}\left(t_{i j k l}-l s g_{l}\right) x_{i j k}-d g_{l}^{+} \leq 0 \quad \forall l \in S
$$

\subsection{Restrições de limite inferior de garantia}

As restrições (11) definem limites mínimos para os parâmetros de qualidade da mistura. 


$$
\frac{\sum_{k \in \text { Patio }} \sum_{i \in \operatorname{Baliza}(k)} \sum_{j \in \text { Patio }} \sum_{i \in \operatorname{Baliza}(k)} t_{i j k l} x_{i j k} x_{i j k}}{j \in \text { Parte }} \geq l i g_{l} \quad \forall l \in S
$$

Linearizando e introduzindo variáveis de desvio negativo de garantia, tem-se:

$$
\sum_{k \in \text { Patio }} \sum_{i \in \text { Baliza }(k)} \sum_{j \in \text { Parte }}\left(t_{i j k l}-\operatorname{lig}_{l}\right) x_{i j k}+d g_{l}^{-} \geq 0 \quad \forall l \in S
$$

\subsection{Restrições de meta}

Visam a penalizar os desvios de quantidade e qualidade em relação aos valores requeridos.

a) Restrições de quantidade:

$$
\sum_{k \in \text { Patio }} \sum_{i \in \operatorname{Baliza}(k)} \sum_{j \in \text { Parte }} x_{i j k}+P^{-}-P^{+}=P_{m} \quad \forall l \in S
$$

b) Restrições de qualidade:

$$
\sum_{k \in \text { Patio }} \sum_{i \in \operatorname{Baliza}(k)} \sum_{j \in \text { Parte }}\left(t_{i j k l}-t r_{l}\right) x_{i j k}+d_{l}^{-}-d_{l}^{+}=0 \forall l \in S
$$

c) Restrições de atendimento aos limites superiores de especificação:

$$
\sum_{k \in \text { Patio }} \sum_{i \in \operatorname{Baliza}(k)} \sum_{j \in \text { Parte }}\left(t_{i j k l}-l s e_{l}\right) x_{i j k}-d e_{l}^{+} \leq 0 \quad \forall l \in S
$$

d) Restrições de atendimento aos limites inferiores de especificação:

$$
\sum_{k \in \text { Patio }} \sum_{i \in \operatorname{Baliza}(k)} \sum_{j \in \text { Parte }}\left(t_{i j k l}-l i e_{l}\right) x_{i j k}+d e_{l}^{-} \geq 0 \quad \forall l \in S
$$

\subsection{Restrições de retomada parcial de uma pilha}

Caso se queira retomar qualquer quantidade $\operatorname{RetParc}_{i j k}$ de minério de uma dada parte $j$ de uma pilha situada em uma baliza $i$ de um pátio $k$, então basta fixar a variável de decisão $x_{i j k}$ nesse valor, isto é:

$$
\begin{aligned}
x_{i j k}= & \operatorname{RetParc}_{i j k} \\
& \forall i \in \operatorname{Baliza}_{(k), \quad \forall k \in \text { Pátio, } \forall j \in \text { Parte, tq }} \\
& \operatorname{RetParc}_{i j k} \neq 0, Q u_{i j k} \neq 0 \text { e Retomar } \\
i j k & \neq 1,
\end{aligned}
$$

\subsection{Restrições de retomada completa de pilhas}

As restrições a seguir fazem com que pilhas previamente definidas pelo operador sejam retomadas completamente.

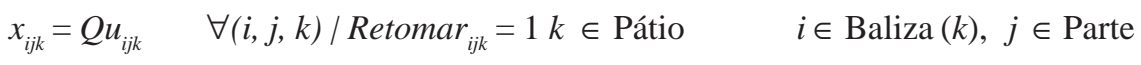

\subsection{Restrições que visam à retomada completa de pilhas}

As restrições a seguir, juntamente com a variável dbalizaijk da função-objetivo, tentam retomar completamente uma pilha visando à disponibilização de espaço no pátio de estocagem.

$x_{i j k}+$ dbaliza $_{i j k}=z_{i j k} \times \quad Q u_{i j k} \quad \forall k \in$ Pátio, $\forall i \in \operatorname{Baliza}(k), \forall j \in$ Parte 


\subsection{Restrições de integralidade e não-negatividade}

As restrições a seguir indicam que as variáveis de decisão assumem valores binários ou não-negativos.

$\begin{array}{ll}\text { dbaliza }_{i j k} \geq 0 & \forall k \in \text { Pátio, } \forall i \in \text { Baliza }(k), \forall j \in \text { Parte } \\ x_{i j k} \geq 0 & \forall k \in \text { Pátio, } \forall i \in \text { Baliza }(k), \forall j \in \text { Parte } \\ z_{i j k} \in\{0,1\} & \forall k \in \text { Pátio, } \forall i \in \operatorname{Baliza}(k), \forall j \in \text { Parte } \\ y_{i j k} \in\{0,1\} & \forall k \in \text { Pátio, } \forall i \in \operatorname{Baliza}(k), \forall j \in \text { Parte } \\ d_{1}^{-} \geq 0 & \forall l \in S \\ d_{1}^{+} \geq 0 & \forall l \in S \\ d e_{1}^{-} \geq 0 & \forall l \in S \\ d e_{1}^{+} \geq 0 & \forall l \in S \\ d g_{1}^{-} \geq 0 & \forall l \in S \\ d g_{1}^{+} \geq 0 & \\ P^{-} \geq 0 & \\ P^{+} \geq 0 & \end{array}$

Observa-se, nesse modelo, que é possível definir, previamente, uma região a ser explorada para a busca de minérios, satisfazendo aos critérios de qualidade e produção. Basta, nesse caso, anular o parâmetro $Q u_{i j k}$ para as regiões que não devem ser exploradas.

\section{Resultados}

O modelo baseado em programação matemática por metas (goal programming) apresentado na seção 4 foi implementado, usando-se o modelador e otimizador LINGO, versão 9.0, da Lindo Systems Inc. (http://www.lindo.com) interfaceando com uma planilha eletrônica do Microsoft Excel.

O sistema desenvolvido foi testado, usando-se 20 instâncias-teste, que consistem em diferentes cenários do Pátio de Controle de Qualidade da Mina de Cauê, em Itabira/MG. Nessas instâncias, são considerados quatro produtos: PCCA, PECA, PXCA_DIPE e PXCA_PPC. Para cada produto, foram analisados seis parâmetros de controle, todos químicos: $\mathrm{Fe}, \mathrm{SiO}_{2}, \mathrm{P}, \mathrm{Al}_{2} \mathrm{O}_{3}$, Mn e $\mathrm{H}_{2} \mathrm{O}$. Cada parâmetro químico l foi classificado em cinco categorias: Irrelevante (-), Importante (IP), Muito Importante (MI), Crítico (CR) e Muito Crítico (CR+), às quais foram associados os seguintes pesos, respectivamente: 0, 1, 5, 10 e 100 . Esses pesos se referem aos desvios de meta $\left(\alpha_{1}^{+}=\alpha_{1}^{-} \forall l \in S\right)$. Os pesos para os desvios de especificação $\left(\gamma_{1}^{+}=\gamma_{1}^{-}\right)$e para os desvios de garantia $\left(\varphi_{1}^{+}=\varphi_{1}^{-}\right)$foram fixados, respectivamente, em 100 e 10000 vezes os pesos para os desvios de meta. Além disso, de forma a tornar comparáveis os desvios de cada parâmetro, os seus respectivos pesos foram multiplicados por 1,10 , $1000,100,100$ e 1 . A penalidade por desvio na meta de produção $\left(\beta^{-}=\beta^{+}\right)$foi fixada em um valor elevado, no caso, um bilhão.

Cada instância foi processada pelo sistema desenvolvido em um microcomputador com processador Celeron, clock de 2,4 GHz, com 256 MB de RAM, demandando um máximo de 30 segundos para encontrar a solução ótima.

A seguir são apresentados dois gráficos típicos, comparando as soluções produzidas pelo sistema com as geradas manualmente pelo operador da empresa de mineração. Em cada gráfico, também são apresentadas as metas de qualidade e os limites de especificação para cada parâmetro de controle. O detalhamento completo dos testes pode ser encontrado em Moraes (2005).

A Figura 1 compara o teor de sílica relativo ao produto PCCA obtido em cinco cenários nas duas abordagens.

A Figura 2 compara o teor de alumina relativo ao produto PXCA_DIPE obtido em cinco cenários nas duas abordagens.

As Figuras 1 e 2 mostram que o sistema desenvolvido produziu soluções significativamente melhores que a solução da empresa, seja atingindo a meta de qualidade (caso da sílica na Figura 1) ou aproximando-se mais dela (caso da alumina na Figura 2). Além disso, o sistema gastou, no pior caso, 60 segundos para encontrar a solução ótima; enquanto o operador gasta, normalmente, de 10 a 15 minutos para encontrar uma única solução, não necessariamente a melhor.

\section{Conclusões}

Esse trabalho apresenta um modelo de programação linear por metas para resolver um problema de blendagem, visando à composição de lotes de minério de ferro da Companhia Vale do Rio Doce, Mina de Cauê. O modelo proposto procura atender as metas de produção e qualidade relativas aos produtos requeridos, respeitando suas especificações e as restrições operacionais dos pátios de minérios.

O sistema desenvolvido foi validado, usando-se um conjunto de 20 cenários reais dos pátios de minérios do complexo Cauê e comparando-se os resultados obtidos com aqueles produzidos manualmente pelos operadores da empresa.

Os resultados obtidos mostraram que o sistema desenvolvido foi capaz de gerar, rapidamente, soluções de qualidade superior às produzidas manualmente, sendo que, em alguns casos, foi capaz de atingir a meta de qualidade para o parâmetro considerado mais crítico, a sílica. Além da melhoria da qualidade da solução gerada e da redução significativa 


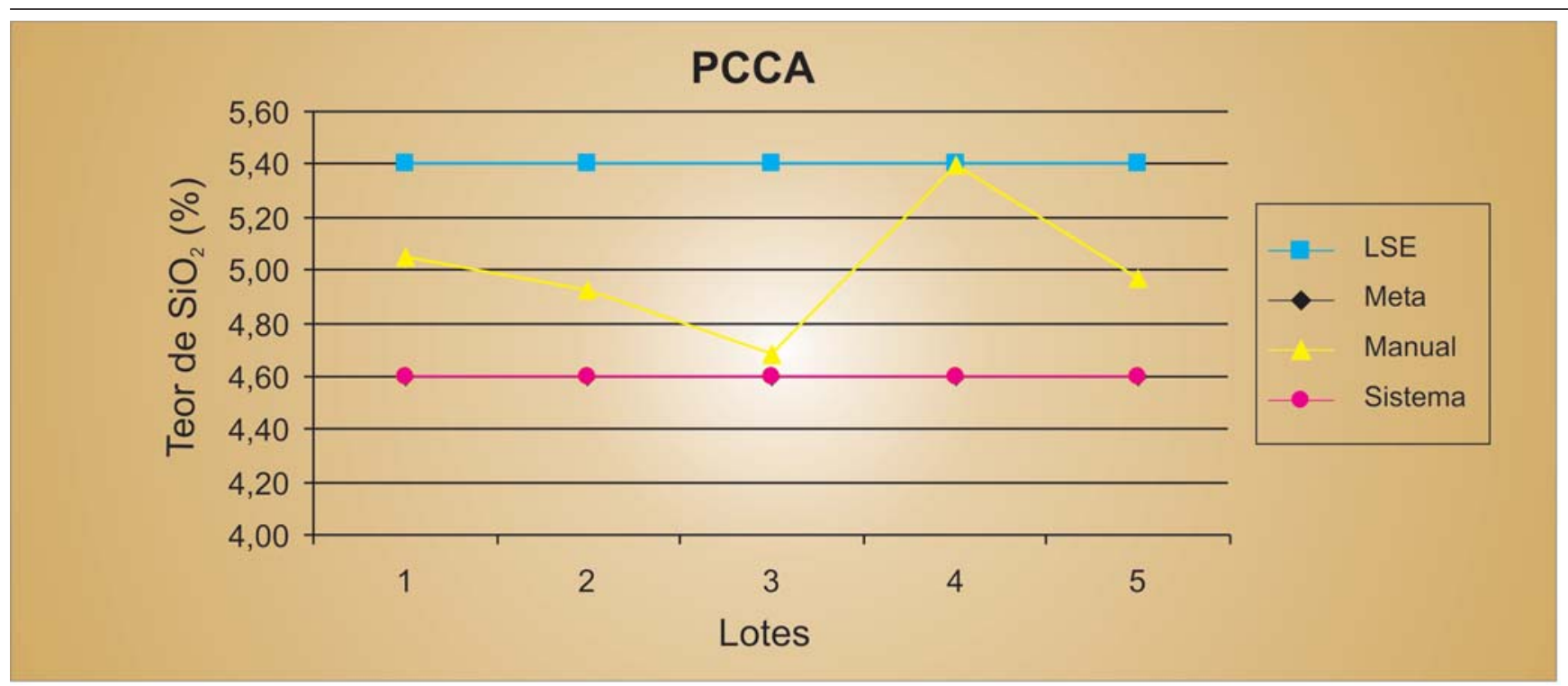

Figura 1 - Teor de sílica em 5 cenários do produto PCCA.

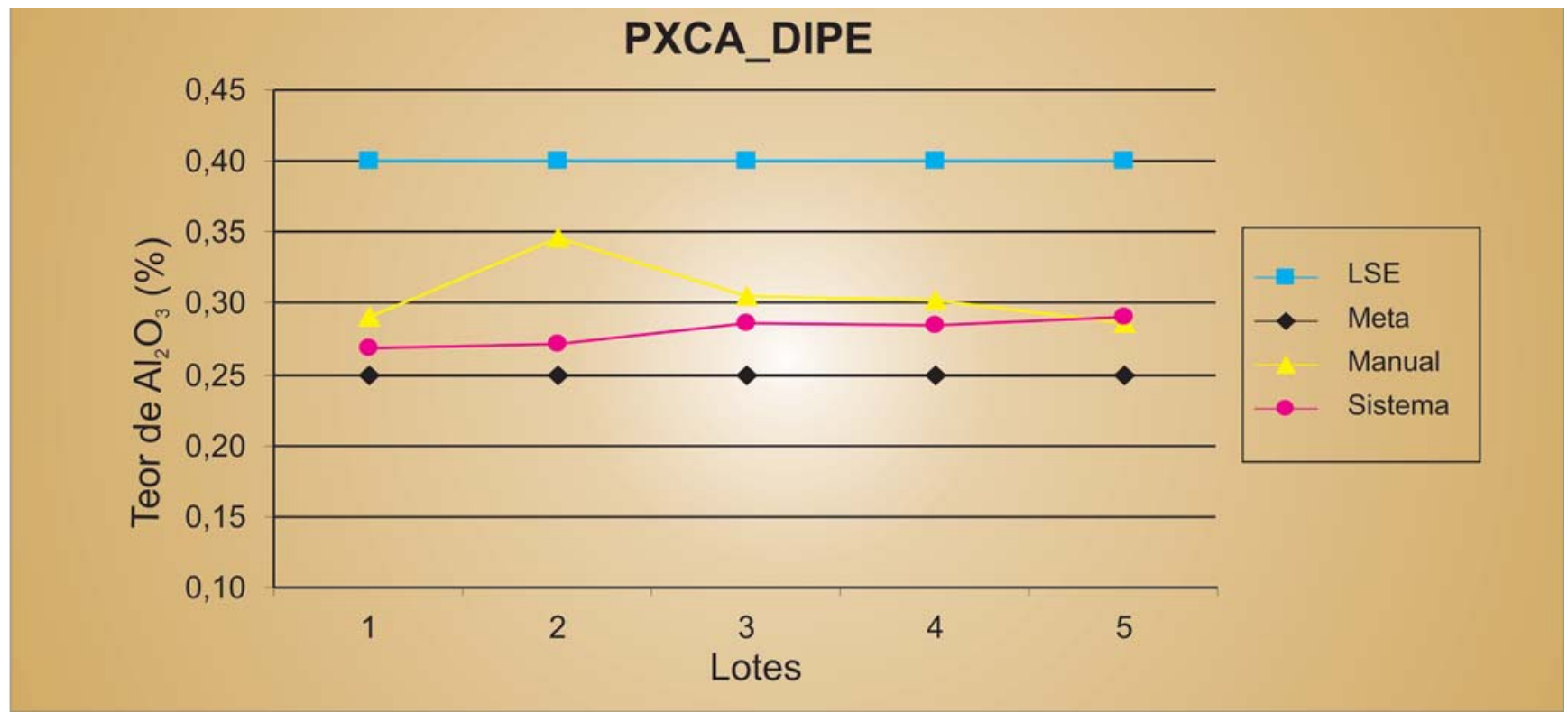

Figura 2 - Teor de alumina em 5 cenários do produto PXCA_DIPE.

no tempo para a tomada de decisão, o sistema confere mais confiabilidade ao processo, pois, ao contrário do ser humano, não é susceptível a erro.

\section{Referências Bibliográficas}

BUENO, A. F., OLIVEIRA, M. C. Goal programming (programação multiobjetivo). In: CORRAR, L. J. e THEÓPHILO, C. R. (Org.). Pesquisa Operacional para decisão em Contabilidade $e$
Administração. São Paulo: Editora Atlas, 2004. Cap. 8, p. 394-438.

CHANDA, E. K. C., DAGDELEN, KADRI. Optimal blending of mine production using goal programming and interactive graphics systems. International Journal of Surface Mining, Reclamation and Environment, v. 9, p. 203-208, 1995.

MORAES, E. F. Um modelo de programação matemática para otimizar a composição de lotes de minério de ferro da Mina Cauê da CVRD. Ouro Preto: Programa de Pós-Graduação em Engenharia Mineral, UFOP, 2005. 89p. (Dissertação de mestrado).
ROMERO, C. A general structure of achievement function for a goal programming model, European Journal of Operational Research, v. 153, p. 675686, 2004.

SCHOFIELD, C. G. Homogenisation/ blending systems design and control for Minerals Processing, Trans Tech Publications, 1980.

CVRD. Estudo de reavaliação de recursos $e$ reservas minerais das minas de Itabira. Itabira: Companhia Vale do Rio Doce, 2003. 39p. (Relatório Técnico).

\section{Artigo recebido em 05/01/2006 e} aprovado em 19/06/2006. 